\title{
Quantifying the relationship between patient characteristics and involvement in developing and implementing a treatment plan
}

This article was published in the following Dove Press journal:

Drug, Healthcare and Patient Safety

16 January 2017

Number of times this article has been viewed

\section{Natalie T Roy \\ Erin E Ulrich}

Department of Pharmaceutical, Biomedical, and Administrative Sciences, College of Pharmacy and Health Sciences, Drake University, Des Moines, IA, USA
Correspondence: Erin E Ulrich

College of Pharmacy and Health Sciences,

Drake University, Fitch Hall II3, 2507

University Avenue, Des Moines, IA

503 I I-4505, USA

Tel + I $5|527| 1846$

Fax + I 5I5 27I I867

Email erin.ulrich@drake.edu
Objective: Improving the patient-physician relationship through patient involvement in the care may lead to improved patient safety and better health outcomes. There exists a gap in knowledge in identifying factors that affect self-reported patient involvement in individualized treatment plans. The objectives of this study were to 1) describe patients' perceptions of their involvement in the creation and implementation of their treatment plans and 2) determine if patient involvement varied by medical condition or demographic characteristics.

Methods: This study was a cross-sectional analysis of data from the "Quality of Care" module of the 2008 Health and Retirement Study (HRS). The individuals of HRS surveys were older than 50 years. One-way analyses of variance were conducted to determine differences between patient characteristics and involvement in creating a treatment plan. A linear regression was conducted to determine predictors of the summed involvement score.

Results: Average summed scores for each domain (shared decision-making, counseling, and follow-up) and overall involvement scores were $\sim 50 \%$. Linear regression showed that being non-White, older age, and diagnosed with a psychiatric condition or diabetes were predictors of increased self-reported involvement in the development and communication of a patient's treatment plan.

Conclusion: Age, race, and having diabetes or a psychiatric condition were the major predictors affecting patient involvement in care, although overall involvement in care was low for all groups. Practice implications: Patient involvement in care was lower than expected and should be further studied to determine the effects of involvement on health outcomes.

Keywords: patient involvement, decision-making, counseling

\section{Introduction}

Quality of care is an important indicator for payment of health care services in the current US health care system. The National Committee for Quality Assurance (NCQA) measures quality using the Healthcare Effectiveness Data and Information Set (HEDIS). ${ }^{1}$ Patient-centered medical homes (PCMHs) are transforming the current health care practice through patient-focused care, including engagement of patients in a team environment to improve health outcomes. ${ }^{2}$ An emerging quality measure is patient satisfaction, although this factor has not yet been studied as extensively as clinical outcomes related to patient health. ${ }^{3,4}$ Studies have shown that patient-centered care, involving an individualized treatment plan, leads to improved health outcomes and better patient satisfaction. ${ }^{5-9}$ One study even researched the extent to which trust between the patient and health care professionals can affect one's health care 
experiences. ${ }^{10}$ Another study found that patient-reported medical errors in diagnosis and treatment were related to the breakdown in the communication between the patient and the clinician. ${ }^{11}$ Improving patient satisfaction with care and the patient-physician relationship may lead to improved patient safety through open communication. However, it is unknown whether patients of varying demographics feel it is important for them to be a part of the health care decisionmaking process for their treatment plan. ${ }^{12}$

Although quality of health care has been studied extensively over the past years as the health care payment system moves to a quality-based incentive system, there is a gap in knowledge in identifying factors that affect self-reported patient satisfaction with patient care, especially as it relates to a patient's involvement in his or her individualized treatment plan, and comparing the experiences between varying demographic groups. This study is timely and innovative because it aims to determine the relationships between patient involvement in care and various factors utilizing a secondary dataset with robust sampling.

\section{Framework}

Literature illustrates that there are four components of developing and implementing a treatment plan. ${ }^{13,14}$ This study evaluated specific questions asked of patients that relate to the four constructs. We also studied patient involvement in the shared decision-making and counseling components of the treatment plan. An overview of this framework is as follows:

1. Clinical evaluation: Utilize a systematic, dynamic process of collecting and analyzing data and interviewing patients to determine a problem list and organize a treatment team.

2. Shared decision-making: Based on the assessment and diagnoses, prioritize problems, create reasonable goals, and determine interventions.

3. Counseling and communication of treatment plan: Discuss areas for concern, create action steps, and provide education to empower patients in managing conditions.

4. Follow-up: Discuss progress, review treatment plan, and document all elements of care plan for effective continuity of care.

The physical examination has been the cornerstone of diagnosis for centuries. Over time, there has been a shift from "bedside diagnosis" to using diagnostic testing to determine a patient's medical conditions or diseases. ${ }^{15}$ Once the diagnoses are made, it is vital that an up-to-date problem list is developed. In addition, the recent Meaningful Use standards advise health care professionals to maintain lists of current and active diagnoses for each patient. ${ }^{16}$ There are varying schools of thought on the importance of an "Annual Physical", but often times the examination becomes as much an act of relationship building and continuity as it is a means of searching for clinically significant findings. ${ }^{17}$

After developing a problem list, the patient and provider can begin to create goals and prioritize the problems. According to the authors of a recent article in the Journal of Atrial Fibrillation on integrated chronic care management for patients with atrial fibrillation, an integral part of the treatment plan is applying evidence-based guidelines to individual patients and providing support when making the clinical decisions. ${ }^{18}$ Thus, in order to achieve successful integrated chronic care management, it is important to incorporate patients and providers in the care process. ${ }^{18}$ Within the relational coordination theory framework, productive patientprofessional interaction occurs through frequent, high-quality communication that is supported by relationships based on shared goals, shared knowledge, and mutual respect. ${ }^{19}$

In the communication component, the health care professionals and patients discuss a strategy to manage the health problem in a way that integrates well with the patient's life. A patient's behavior change becomes the focus of the care plan and the patient cannot see him/herself as a passive recipient of heath care services. What patients do for themselves on a daily basis influences their health far more than medical interventions alone, according to the World Health Organization (WHO).$^{20}$ Therefore, providing adequate education and support to patients during this phase is important in managing their chronic condition. However, low health literacy and lack of understanding in decision-making may inhibit a patient's involvement. Thus, utilizing a variety of educational methods can allow patients to play a more active role in their treatment. ${ }^{21}$

Finally, follow-up is an important part of the treatment plan in order to drive improvement in health and positive reinforcement. ${ }^{22}$ This also contributes to the "continuum of care", rather than individual episodes or health care visits. ${ }^{22}$ Having multiple quality interactions and an open communication line lends for more patient involvement, although the patient-professional interaction is not solely effective in protecting against the deterioration of self-management abilities in some groups of chronically ill patients. ${ }^{19}$

For this study, we focus on patient involvement in the last three constructs: shared decision-making, counseling and communication, and follow-up. Figure 1 presents this treatment plan framework with examples that fall within each of the care components. 


Clinical evaluation
- Interview patient
- Diagnostic summary
- Problem list

Figure I Framework for treatment plan development and communication.

\section{Objectives}

The first objective of this study was to determine patients' self-reported level of involvement in the creation and implementation of their treatment plan. Drawing from past literature, we hypothesized that the majority of patients have overall poor feelings toward their involvement in their treatment plan. The second objective was to determine if the level of involvement varied by condition. We hypothesized that those who had symptomatic conditions that required a significant amount of self-care and monitoring would be more involved in shared decision-making of their treatment plan. Those who have a condition that impacts their daily quality of life may have more desire to have a voice in what treatment is selected and how it would be implemented.

\section{Methods Design}

This study used a cross-sectional analysis of data from the Experimental Module "Quality of Care" of the 2008 Health and Retirement Study (HRS). Although the HRS does include newer core data each year, quality of care data is found only in this experimental module and has never been analyzed to measure patient involvement in treatment plan development and implementation. The study was approved by the institutional review board of Drake University in June 2015.

\section{Data sources HRS}

The University of Michigan HRS is a longitudinal panel study that biennially surveys a representative sample of $>20,000$ Americans older than 50 years to explore the changes in labor force participation and health transitions that older Americans undergo. Since 1992, the study has collected information about income, work, assets, pension plans, health insurance, disability, physical health and functioning, cognitive functioning, and health care expenditures. The HRS is sponsored by the National Institute on Aging (grant number NIA U01AG009740) and the Social Security Administration and is conducted by the University of Michigan Institute for Social Research. This study focuses on the 2008 HRS panel. The data collection period for the 2008 interview was February 2008-February 2009.

\section{Quality of care HRS experimental module}

A random subsample of the HRS respondents was selected and asked to complete the quality of care model after the standard HRS survey. This module included items focused on patients' self-report of their experience around their treatment of one of the following conditions: pain, lung disease, emotional/nervous/psychiatric problem, diabetes, heart problem, high blood pressure, or arthritis. The diagnosis code reported in the HRS core survey was used to determine if the respondents were eligible for this section. If eligible, this HRS diagnosis code was also used to determine which condition the respondent would be referring to when answering questions.

\section{Study population}

The inclusion criteria consisted of subjects who completed the 2008 HRS and the Quality of Care HRS Experimental Module. This sample included Americans aged 50 years or older. Written informed consent was obtained during initial data collections by HRS.

\section{Measures of interest}

All items in the HRS Quality of Care Experimental Module were on a 5-point Likert scale (almost never, generally not, sometimes, most of the time, and almost always). Respondents could also report "don't know" or "refuse to answer" and were excluded from the analyses. All questions in this module were asked about their care related to a specific condition, designated at the beginning of the module, within the past 6 months. All questions were asked in the form of "When you received care for your [condition] over the past 6 months, how often were you ?". The HRS Quality of Care Experimental Module contains 13 items that fall within the four constructs of an effective treatment 
plan. There has been no previous work with these involvement items. From the HRS dataset, items were selected that aligned with theory and past literature.

\section{Construct 2: shared decision-making}

The second construct included four items, all of which captured the respondents' involvement with their shared decision-making. These questions included: 1) "given choices about treatment to think about?"; 2) "asked for your ideas when you made a treatment plan?"; 3) "asked to talk about your goals in caring for your illness?"; and 4) "sure that your doctor or nurse thought about your values and your traditions when they recommended treatments to you?".

\section{Construct 3: counseling and communication}

This construct included three items that captured the respondents' involvement in integrating the health decisions into their life. These questions included: 1) "helped to set goals to improve your eating or exercise?"; 2) "helped to make a treatment plan that you could do in your daily life?"; and 3) "helped to plan ahead so you could take care of your illness even in hard times?".

\section{Construct 4: follow-up}

This construct included two items related to patient followup. These questions were 1) "contacted after a visit to see how things were going?" and 2) "shown how what you did to take care of your [condition] influenced your condition?".

\section{Demographics}

The interviewer documented the subject's gender and coded it as a dummy variable with females being the reference group. The level of education attained had the following response options: no degree, General Educational Development (GED), high school diploma, 2-year college, 4-year college, master degree, professional degree, and degree unknown/some college. Education was categorized into three groups, less than high school, high school diploma, and at least a 4-year degree. Race response options included White/ Caucasian, Black/African American, and others. Ethnicity was grouped into two, Hispanic or non-Hispanic.

\section{Rural-urban status}

Beale Codes (also known as Rural-Urban Continuum Codes) were developed by the United States Department of Agriculture (USDA) and categorize populations into 12 groups by size. The HRS groups the 12 Beale Codes into three categories, urban, suburban, and exurban.

\section{Geographic region}

HRS codes geographic regions of the US into 13 groups. For this study, HRS geographic codes were then further categorized into Northeast, Midwest, South, and West.

\section{Analyses}

The results were analyzed using SPSS 22.0 (IBM Corporation, Armonk, NY, USA). Overall frequencies and descriptive statistics were conducted on all items. Chi-square tests were conducted to explore the differences between condition, demographics, and each involvement item. A summed involvement score was created for each of the three domains (shared decision-making, counseling, and follow-up). In addition, a total summed score of patient involvement was created with a range from 8 (not involved) to 40 (completely involved). One-way analyses of variance (ANOVAs) were conducted to explore the differences between conditions and demographics on total involvement in creation and implementation of a treatment plan. A linear regression was conducted with all measures of interest to determine predictors of summed involvement score. Internal consistency reliability was determined for the eight patient involvement items.

\section{Results}

The majority of the respondents were female, were White, received a high school diploma, had an average of 2.61 conditions, and were an average the age of 73 years (Table 1). Subjects were most commonly living in the South or Midwest and in an urban area (Table 1). The two most common conditions reported by respondents were arthritis and heart condition (Table 1).

Subjects were assigned a medical condition they were previously diagnosed with in order to answer care questions related to that condition. This decreased the sample size from 1,572 to 890 due to 682 subjects reporting having multiple comorbidities. Average scores ranged from 2.16 to 3.21 out of 5 for each item (Table 2). Items were summed within each domain, revealing average scores for shared decision-making, counseling, and follow-up to be 10.16 out of a possible 20 $(\mathrm{SD}=4.2), 4.89$ out of a possible of $10(\mathrm{SD}=2.54), 5.22$ out of a possible $10(\mathrm{SD}=2.37)$, respectively. Summed patient involvement scores were computed. An average total patient involvement score was $\sim 20$ out of a total 40 (Table 2). There were three statistically significant one-way ANOVAs. Those who were non-White reported having higher involvement in the development and implementation of their treatment plan (Table 3). Subjects who reported being diagnosed with a psychiatric condition or diabetes were more likely to report 
Table I Overall sample demographics

\begin{tabular}{ll}
\hline Characteristic & Sample (n = I,572), n (\%) \\
\hline Gender & $608(38.7)$ \\
Male & $964(61.3)$ \\
Female & \\
Race/ethnicity & $1,307(83.1)$ \\
White/Caucasian & $224(14.2)$ \\
Black/African American & $41(2.6)$ \\
Other & $153(9.7)$ \\
Hispanic & \\
Education level attained & $359(22.8)$ \\
No degree & $917(58.3)$ \\
GED/high school diploma & $296(18.8)$ \\
At least a 4-year degree & \\
Geographic region 2006 & $269(17.1)$ \\
Northeast & $389(24.7)$ \\
Midwest & $291(18.5)$ \\
West & $619(39.4)$ \\
South & \\
Urban/rural 2006 & $738(46.9)$ \\
Urban & $314(20.0)$ \\
Suburban & $516(32.8)$ \\
Exurban & \\
Condition & $196(12.5)$ \\
Lung disease & $296(18.8)$ \\
Emotional/psychiatric problem & $286(18.2)$ \\
Diabetes & $497(31.6)$ \\
Heart problem & $374(23.8)$ \\
High blood pressure & $1,155(73.5)$ \\
Arthritis & $296(18.8)$ \\
Cancer & $134(8.5)$ \\
Stroke & Mean (SD) \\
Age, years & $73.62(10.75)$ \\
Number of comorbidities & $2.61(1.33)$ \\
\hline Abbrviation: GED, Gener & \\
\hline &
\end{tabular}

Abbreviation: GED, General Educational Development.

Table 2 Overall sample care and involvement

\begin{tabular}{ll}
\hline Activity & $\begin{array}{l}\text { Sample }(\mathbf{n}=\mathbf{8 9 0}), \\
\text { mean (SD) }\end{array}$ \\
\hline $\begin{array}{l}\text { Shared decision-making } \\
\quad \text { Given choices about treatment to think about }\end{array}$ & $2.28(\mathrm{I} .40)$ \\
$\begin{array}{l}\text { Asked for your ideas when you made a } \\
\text { treatment plan }\end{array}$ & $2.24(\mathrm{I} .45)$ \\
$\begin{array}{l}\text { Asked to talk about your goals in caring for } \\
\text { your illness }\end{array}$ & $2.43(\mathrm{I} .54)$ \\
$\begin{array}{l}\text { Your values and traditions were taken } \\
\text { into consideration when they recommend }\end{array}$ & $3.2 \mathrm{I}(\mathrm{I} .60)$ \\
$\begin{array}{l}\text { treatments to you } \\
\text { Counseling on treatment plan }\end{array}$ & \\
$\begin{array}{l}\text { Helped to set goals to improve your eating and } \\
\text { exercise }\end{array}$ & $2.65(\mathrm{I} .50)$ \\
$\begin{array}{l}\text { Helped to plan ahead so you could take care of } \\
\text { your illness even in hard times }\end{array}$ & $2.24(\mathrm{I} .50)$ \\
$\begin{array}{l}\text { Follow-up } \\
\text { Contacted after visit to see how things are going }\end{array}$ & $2.16(\mathrm{I} .4 \mathrm{I})$ \\
$\begin{array}{l}\text { Shown how what you did to take care of your } \\
\text { condition influenced your condition/health }\end{array}$ & $3.06(\mathrm{I} .52)$ \\
\begin{tabular}{l} 
Summed involvement score (8-40) \\
\hline
\end{tabular} & $20.80(8.44)$ \\
\hline
\end{tabular}

Table 3 Significant one-way ANOVA results between subject characteristics and summed patient involvement score

\begin{tabular}{llllll}
\hline Characteristic & $\boldsymbol{n}$ & Mean & SD & $\boldsymbol{F}$ & $\boldsymbol{p}$-value \\
\hline Race & & & & 8.02 & 0.005 \\
$\quad$ White & 628 & 22.42 & 9.10 & & \\
$\quad$ Black & 146 & 24.80 & 9.34 & & \\
$\quad$ Others & 21 & 26.38 & 9.20 & & \\
$\begin{array}{l}\text { Psychiatric condition } \\
\quad \text { Yes }\end{array}$ & 106 & 24.74 & 10.07 & & \\
$\quad$ No & 689 & 22.70 & 9.04 & & \\
$\begin{array}{l}\text { Diabetes } \\
\quad \text { Yes }\end{array}$ & 216 & 24.37 & 9.82 & & \\
$\quad$ No & 579 & 22.44 & 8.91 & & \\
\hline
\end{tabular}

Abbreviation: ANOVA, analysis of variance.

more involvement than those who reported having any other condition (Table 3).

A linear regression was conducted with all demographics and measures of interest found in Table 1 to determine which variables were strong predictors of summed involvement score. Dummy variables were created for education, geographic region, and urban/rural status. Race was further categorized into White and non-White. Although this full model was statistically significant $(F=2.40, p=0.001)$ and explained $\sim 33 \%$ of patient involvement, only three variables were significant at predicting patient involvement score: age $(\beta=-0.131, p<0.001)$, race $(\beta=2.10, p=0.022)$, and answering the care questions about their diabetes treatment ( $\beta=1.51, p=0.047)$. A partial model was run including control variables and the statistically significant variables in the full regression model. This partial model included age, gender, race, ethnicity, education, and answering care questions about diabetes treatment. This partial model was statistically significant $(F=5.68, p<0.001)$ and explained $\sim 40 \%$ of patient involvement (adjusted $R^{2}=0.41$ ). The same three variables were significant predictors: age $(\beta=-0.139$, $p<0.001)$, race $(\beta=2.174, p=0.013)$, and responding to the care questions about their diabetes treatment $(\beta=1.49$, $p=0.046$ ). The patient involvement items were highly reliable (eight items; $\alpha=0.837$ ).

\section{Discussion and conclusion Discussion}

Quality of care is an important indicator for payment of health care services in the US and has been a focus for many health organizations in recent years. Measuring patient satisfaction is a modern way of evaluating quality of care in addition to clinical outcomes of patient health. Thus, it is important to begin identifying factors that affect self-reported patient satisfaction with their care. It is predicted that patients' 
involvement in their care would affect overall satisfaction, so this study determined the level of patient involvement in an individualized treatment plan and compared the experiences between medical conditions and varying demographic groups. This research can drive changes in patients' involvement in their health care in order to improve overall quality of care and health outcomes.

The first finding from this study was that the overall summed patient involvement score was unexpectedly low with a range from 2.16 to 3.21 out of 5 for each item relating to involvement on the questionnaire. The average total involvement scores were only 20 out of a total 40 . These low summed involvement scores likely indicate that patients are "generally not" or only "sometimes" involved in their health care relating to a particular medical condition. Most of the existing literature on this topic is more qualitative in nature and studied individual patient's preferences with the patient's involvement in care and effectiveness of specific techniques. ${ }^{23-25}$ This is an important finding because now that we know that patients are not very involved in their health care, we can further study how this lack of involvement affects quality of care, health outcomes, and patient safety. Because the summed involvement score took into account shared decision-making, counseling and communication of treatment plan, and follow-up, physicians could better emphasize each of these areas in the patient's treatment plan. This would require a collaborative effort between health systems and individual health care professionals.

This study found a significant difference in involvement between patients with diabetes and patients without diabetes. Patients with diabetes reported having higher involvement in their health care (summed involvement score 24.37/40) than patients without diabetes (summed involvement score 22.44/40). Previous literature has not compared involvement in health care between patients with diabetes and without diabetes. Most of the existing literature studied only patients with diabetes and the impact that involvement has on patients' health outcomes. ${ }^{23,24}$ These results may indicate that patients with diabetes may be more involved in their care due to the nature of the disease state and needing to incorporate their treatment plan into all aspects of their lives. This finding will be important for physicians and other health care professionals to keep in mind when working collaboratively with patients with diabetes.

There was also a significant difference in involvement between patients with psychiatric conditions and patients without psychiatric conditions. Patients with a psychiatric condition reported having higher involvement in their health care (summed involvement score 24.74/40) than patients without a psychiatric condition (summed involvement score 22.70/40). Similar to the diabetes literature, there has not been a comparison of patients with a psychiatric condition to patients without a psychiatric condition. However, existing literature has examined the value of patients' involvement in their psychiatric condition and has found that involvement needs to be individualized due to the variable nature of psychiatric conditions. ${ }^{25-27}$ Health care professionals working with patients with psychiatric conditions will likely require more patient involvement in order to achieve better health outcomes and better patient satisfaction.

The linear regression showed that age, race, and having diabetes were the only statistically significant predictors of the summed involvement score. A study by Cramm and Nieboer ${ }^{19}$ specifically on self-management of chronic conditions found a negative relationship between older age and self-management, which is consistent with our results that older age is related to decreased involvement in health decision-making. As for race, most past literature has examined communication between patients and providers to determine racial/ethnic health disparities versus actually comparing involvement of patients of differing races. ${ }^{28,29}$ This will be an important factor to analyze in future studies in order to determine the benefits of more patients' involvement in their care as it relates to race.

\section{Limitations}

There were four limitations to this study. In designing this study, we were limited on how to conceptualize patient involvement in health care. The questions that we utilized came from a pre-existing dataset, so we were not able to ask direct questions to patients about their involvement; rather, we inferred from the selected questions the involvement that patients had through the frequency of the actions described in the questionnaire. However, utilizing a framework supported by past literature assists in strengthening the items we selected from the secondary dataset. Other studies have utilized focus groups, open-ended questions, and face-toface interviews to assess patient involvement ${ }^{30-32}$ - none of which are practical as a part of a longitudinal panel study. In future studies, we hope to utilize the Control Preferences Scale or the Decisional Conflict Scale ${ }^{33}$ to measure patient involvement on a deeper level. However, we do believe that the range of questions asked in the Quality of Care Experimental Module does address patient involvement in a way unique from previous literature.

Second, as in all survey research, recall bias is a limitation that likely affected our study. Participants were asked to recall 
how often a particular health care-related event or interaction occurred in the past 6 months. Recall of this information depends entirely on a person's memory, which is often imperfect and unreliable. Thus, we understand that these self-reported data provide a threat to the internal validity of the study.

The third limitation may be the short duration of the recall time asked of participants. Each question focused on a health care-related event or interaction pertaining to a particular medical condition in the past 6 months. This could cause a recall bias if the patient had not been involved in his or her care for that particular condition in only the past 6 months and yet was involved prior to 6 months ago. The positive aspect of this limitation is that 6 months is a relatively short time for a participant to remember, rather than a year or more from the time the questions were asked.

The last limitation is the generalizability of these findings. The HRS provides nationally representative weights. However, according to the Institute for Social Research, these weights cannot be applied to the experimental modules. This study utilized the Quality of Care Experimental Module. Therefore, findings from this study must be generalized cautiously.

\section{Conclusion}

The purpose of this study was to identify factors that affect self-reported patients' involvement in their health care and compare experiences between varying demographic groups. We found that age, race, and having diabetes were the three major predictors affecting patient involvement in care. However, overall, patients reported relatively low involvement in shared decision-making, counseling and communication of treatment plan, and follow-up, which resulted in an overall low summed involvement score. These data will be useful in determining future improvements in involving patients in their health care and whether these improvements also improve quality of care, health outcomes, and patient safety.

\section{Practice implications}

Overall, we found that patients self-reported being $50 \%$ involved in their treatment plan. This is an unfortunately shocking finding, considering that patient involvement likely affects patient satisfaction, quality of care, and patient safety. Because quality of care is such an important indicator for payment of health care services in the current health care system, this is clearly an area that needs to be further studied in order to determine factors related to lack of involvement. Our study found four predictors that affect patient involvement, including race, age, psychiatric condition, and diabetes. In future studies, these specific predictors could be studied to determine appropriate ways to better involve these patient populations in their care. We can also study how patient involvement affects patient safety (adverse drug events, access to services, and medication errors) and work to improve patient safety through improved patient involvement in the treatment plan. However, knowing that patients are potentially less involved in their care than expected, health systems and health care providers may need to reevaluate their processes and procedures to better involve patients in their health decisions and treatment plans.

\section{Acknowledgments}

Allison Tenhouse, a research assistant at the time, assisted in manuscript preparation and revisions. No funding was required for this project. There has been no prior presentation of this work.

\section{Author contributions}

All authors contributed toward data analysis, drafting and critically revising the paper and agree to be accountable for all aspects of the work.

\section{Disclosure}

The authors report no conflicts of interest in this work.

\section{References}

1. National Committee for Quality Assurance [webpage on the Internet] HEDIS Measures. National Committee for Quality Assurance. Available from: http://www.ncqa.org/HEDISQualityMeasurement/HEDISMea sures.aspx. Accessed June 2015.

2. National Committee for Quality Assurance. The Future of PatientCentered Medical Homes. National Committee for Quality Assurance. Available from: http://www.ncqa.org/Portals/0/Public\%20 Policy/2014\%20Comment\%20Letters/The_Future_of_PCMH.pdf. Accessed September 2015.

3. Swinglehurst D, Emmerich N, Maybin J, Park S, Quilligan S. Confronting the quality paradox: towards new characterisations of 'quality' in contemporary healthcare. BMC Health Serv Res. 2015;15(1):240.

4. Harper JL, De costa AM, Garrett-Mayer E, Sterba KR. Incorporating patient satisfaction metrics in assessing multidisciplinary breast cancer care quality. South Med J. 2015;108(6):372-376.

5. Sharp S, McAllister N, Broadbent M. The vital blend of clinical competence and compassion: how patients experience person-centered care Contemp Nurse. 2016;52(2-3):300-312.

6. Hird AE, Lemke M, Turovsky M, et al. Doctor, what are my options? A prospective cohort study of an individualized care plan for patients with gastrointestinal cancer. Curr Oncol. 2015;22(3):e171-e177.

7. Dubois S, Loiselle CG. Cancer informational support and health care service use among individuals newly diagnosed: a mixed methods approach. J Eval Clin Pract. 2009;15(2):346-359.

8. Gunadi S, Upfield S, Pham ND, Yea J, Schmiedeberg MB, Stahmer GD. Development of a collaborative transitions-of-care program for heart failure patients. Am J Health Syst Pharm. 2015;72(13):1147-1152.

9. Fischer M, Visser A, Voerman B, Garssen B, van Andel G, Bensing J. Treatment decision making in prostate cancer: patients' participation in complex decisions. Patient Educ Couns. 2006;63:308-313.

10. Cohn S. 'Trust my doctor, trust my pancreas': trust as an emergent quality of social practice. Philos Ethics Humanit Med. 2015;10(1):9. 
11. Kuzel A, Woolf S, Gilchrist V, et al. Patient reports of preventable problems and harms in primary health care. Ann Fam Med. 2004;2(4): 333-340.

12. Renedo A, Marston C. Developing patient-centered care: an ethnographic study of patient perceptions and influence on quality improvement. BMC Health Serv Res. 2015;15:122.

13. Hutchinson M, Casper P, Harris J, et al. The Clinician's Guide to Writing Treatment Plans and Progress Notes. Available from: https://www. sccgov.org/sites/dads/Adult\%20System\%20of\%20Care\%20Policy\%20$\% 20$ Procedure/Documents/Clinician_Gde_toolkit.pdf. Accessed October 2015.

14. American Nurses Association [webpage on the Internet]. The Nursing Process. American Nurses Association. Available from: http://www. nursingworld.org/EspeciallyForYou/What-is-Nursing/Tools-You-Need/ Thenursingprocess.html. Accessed October 2015.

15. Verghese A, Charlton B, Cotter B, Kugler J. A history of physical examination texts and the conception of bedside diagnosis. Trans Am Clin Climatol Assoc. 2011;122:290-311.

16. Centers for Medicare and Medicaid. Eligible Professional Meaningful Use Core Measures, Measure 3 of 13. Centers for Medicare and Medicaid. Available from: https://www.cms.gov/Regulations-and-Guidance/ Legislation/EHRIncentivePrograms/downloads/3_Maintain_Problem_ ListEP.pdf. Accessed October 2015.

17. Goroll AH. Toward trusting therapeutic relationships - in favor of the annual physical. $N$ Engl J Med. 2015;373:1487-1489.

18. Hendriks JML, Crijn HJGM, Vrijhoef HJM. Integrated chronic care management for patients with atrial fibrillation: a rationale for redesigning atrial fibrillation care. J Atr Fibrillation. 2015;7:45-50.

19. Cramm JM, Nieboer AP. Chronically ill patients' self-management abilities to maintain overall well-being: what is needed to take the next step in the primary care setting? BMC Fam Pract. 2015;16:123.

20. World Health Organization. Innovative Care for Chronic Conditions: Building Blocks for Action. World Health Organization; 2002. Available from: http://www.who.int/chp/knowledge/publications/icccglobalreport.pdf?ua=1. Accessed October 2015.

21. Harter M, Simon D. Do patients want shared decision making and how is this measured? In: Gigerenzer G, Muir JA, editors. Better Doctors, Better Patients, Better Decisions: Envisioning Health Care 2020. Cambridge, MA: MIT Press; 2011:53-58.
22. Scaletta T. Best Practices for Improving Care with Patient Followup. Hospital Impact. Available from: http://www.fiercehealthcare. $\mathrm{com} /$ healthcare/best-practices-for-improving-care-patient-follow-up. Accessed October 2015.

23. Shortus T, Kemp L, Mckenzie S, Harris M. 'Managing patient involvement's: provider perspectives on diabetes decision-making. Health Expect. 2013;16(2):189-198.

24. Vahdat S, Hamzehgardeshi L, Hessam S, Hamzehgardeshi Z. Patient involvement in health care decision making: a review. Iran Red Crescent Med J. 2014;16(1):e12454.

25. Goss C, Moretti F, Mazzi MA, Del Piccolo L, Rimondini M, Zimmermann C. Involving patients in decisions during psychiatric consultations. Br J Psychiatry. 2008;193(5):416-421.

26. Beitinger R, Kissling W, Hamann J. Trends and perspectives of shared decision-making in schizophrenia and related disorders. Curr Opin Psychiatry. 2014;27(3):222-229.

27. Goossensen A, Zijlstra P, Koopmanschap M. Measuring shared decision making processes in psychiatry: skills versus patient satisfaction. Patient Educ Couns. 2007;67(1-2):50-56.

28. Johnson RL, Roter D, Powe NR, Cooper LA. Patient race/ethnicity and quality of patient-physician communication during medical visits. $\mathrm{Am}$ J Public Health. 2004;94(12):2084-2090.

29. Gordon HS, Street RL, Sharf BF, Souchek J. Racial differences in doctors' information-giving and patients' participation. Cancer. 2006;107(6):1313-1320.

30. Carlsson C, Nilbert M, Nilsson K. Patients' involvement in improving cancer care: experiences in three years of collaboration between members of patient associations and health care professionals. Patient Educ Couns. 2006;61(1):65-71.

31. Forbat L, Cayless S, Knighting K, Cornwell J, Kearney N. Engaging patients in health care: an empirical study of the role of engagement on attitudes and action. Patient Educ Couns. 2009;74(1):84-90.

32. Bastiaens H, Van Royen P, Pavlic DR, Raposo V, Baker R. Older people's preferences for involvement in their own care: a qualitative study in primary health care in 11 European countries. Patient Educ Couns. 2007;68(1):33-42.

33. Kremer H, Ironson G, Schneiderman N, Hautzinger M. "It's my body": does patient involvement in decision making reduce decisional conflict? Med Decis Making. 2007;27(5):522-532.
Drug, Healthcare and Patient Safety

\section{Publish your work in this journal}

Drug, Healthcare and Patient Safety is an international, peer-reviewed open access journal exploring patient safety issues in the healthcare continuum from diagnostic and screening interventions through to treatment, drug therapy and surgery. The journal is characterized by the rapid reporting of reviews, original research, clinical, epidemiological and

\section{Dovepress}

post-marketing surveillance studies, risk management, health literacy and educational programs across all areas of healthcare delivery. The manuscript management system is completely online and includes a very quick and fair peer-review system. Visit http://www.dovepress.com/ testimonials.php to read real quotes from published authors. 